\title{
GROUP-INVARIANT SOLUTIONS OF A NONLINEAR ACOUSTICS MODEL
}

\author{
J.C. NDOGMO
}

\begin{abstract}
Based on a recent classification of subalgebras of the symmetry algebra of the Zabolotskaya-Khokhlov equation, all similarity reductions of this equation into ordinary differential equations are obtained. Large classes of group-invariant solutions of the equation are also determined, and some properties of the reduced equations and exact solutions are discussed.
\end{abstract}

\section{INTRODUCTION}

The Zabolotskaya-Khokhlov equation is a popular model for the propagation of nearly plane, weakly nonlinear sound waves, derived from the Navier-Stokes equation [1. Some of the assumptions underlying the derivation of this equation concern the flow, which is isentropic, meaning that the pressure is a function of the fluid density alone, the small viscosity of the flow, and the weak effects of nonlinearity. The general form of the $(2+1)$-dimensional version of this equation can be put in the form

$$
\left(u_{t}+\alpha_{0} u u_{x}\right)_{x}+\alpha_{1} u_{y y}=0 .
$$

In (1.1), $u$ is a scalar representing the gas speed (amplitude disturbances), $y$ is a spatial variable associated with transverse variations, $x$ is a spatial variable associated with variations in the direction of propagation, and $t$ is a time-like variable. The constant $\alpha_{0}$ is a nonlinearity parameter, while $\alpha_{1}$ is a constant parameter associated with diffraction. By a scaling of the independent variables, (1.1) can be put in the normalized form

$$
\Delta(t, x, y, u) \equiv u_{x t}-\left(u u_{x}\right)_{x}-u_{y y}=0 .
$$

The Zabolotskaya-Khokhlov (ZK) equation (1.1) obtained in 1969 was later generalized in a paper by Kuznetsov [2] in 1971 to also account for dissipation in unlimited spaces. The resulting equation is referred to as the Khokhlov-ZabolotskayaKuznetsov (KZK) equation, and has the form

$$
\left(u_{t}-\alpha_{0} u u_{x}-\beta u_{x x}\right)_{x}-\alpha_{1} u_{y y}=0,
$$

where the constant $\beta$ is the dissipation parameter. The ZK equation has been studied from the Lie group approach in a number of papers in which similarity [3, [4, 5] and other types of reductions [6] were obtained. The KZK equation has also been studied for exact solutions in a number of papers. In particular, a Painlevé analysis of this equation was proposed in 7, while a symmetry analysis of the same equation has been undertaken in [8, 9], and for a variant with a variable coefficient $\alpha_{1}$ in [10].

However, as far as reductions to ordinary differential equations (ODEs) are concerned, all the results obtained in these papers were severely limited by a number 
of anzatz, and generally by the lack of classification into subalgebras of the symmetry algebra of the equation. An exception is the KZK variant with a variable coefficient discussed in [10, where the determination of exact solutions is based on a symmetry algebra classification. Although a complete classification of the symmetry algebra of the ZK equation was recently given in [5], this only led in that paper, due to space limitations, to the determination of all similarity reductions to $(1+1)$-dimensional models of the equation.

In this paper, we consider the $(2+1)$-dimensional version of the ZK equation given by (1.2), and using the complete classification of two-dimensional subalgebras of the symmetry algebra of this equation proposed in [5, all similarity reductions to ODEs are obtained. These ODEs have the advantage of being much easier to solve compared to the $(1+1)$-dimensional models obtained in similarity reductions by onedimensional subalgebras, and thus new exact similarity solutions are obtained, and some of their properties are discussed. It is shown in particular that all nonlinear reduced ODEs are essentially non linearizable. Because of their importance in underwater acoustics, in medicine, and in other industries, bounded sound beams solutions of the ZK equation are also exhibited. For the sake of completeness, we also include reductions to $(1+1)$-dimensional models of the equation based on the classification of one-dimensional subalgebras of the ZK symmetry algebra, already obtained in [5].

\section{Classification OF LOW-Dimensional SUBALGEBRAS}

We wish to recall in this section a result on the classification of low-dimensional subalgebras of the symmetry algebra of the ZK equation obtained in [5]. The Lie symmetry algebra $L$ of the ZK equation itself is well-known [11, 12. It is an infinite dimensional algebra with generators

$$
\begin{aligned}
\mathbf{v}_{0} & =2 x \partial_{x}+y \partial_{y}+2 u \partial_{u} \\
\mathbf{x}(g) & =g \partial_{x}-g^{\prime} \partial_{u} \\
\mathbf{y}(h) & =\frac{1}{2} y h^{\prime} \partial_{x}+h \partial_{y}-\frac{1}{2} y h^{\prime \prime} \partial_{u} \\
\mathbf{z}(f) & =f \partial_{t}+\frac{1}{6}\left(2 x f^{\prime}+y^{2} f^{\prime \prime}\right) \partial_{x}+\frac{2 y}{3} f^{\prime} \partial_{y}+\frac{1}{6}\left(-4 u f^{\prime}-2 x f^{\prime \prime}-y^{2} f^{\prime \prime \prime}\right) \partial_{u}
\end{aligned}
$$

where $f, g, h$ are arbitrary functions of the time variable $t$, and where a prime represents a derivative with respect to $t$. It is easy to see that for the Lie algebra $L$, the commutation relations are given by

$$
\begin{aligned}
{\left[\mathbf{v}_{0}, \mathbf{x}(g)\right] } & =-2 \mathbf{x}(g), & {[\mathbf{x}(g), \mathbf{y}(h)] } & =0 \\
{\left[\mathbf{v}_{0}, \mathbf{y}(h)\right] } & =-\mathbf{y}(h), & {[\mathbf{x}(g), \mathbf{z}(f)] } & =\mathbf{x}\left(f^{\prime} g / 3-f g^{\prime}\right) \\
{\left[\mathbf{v}_{0}, \mathbf{z}(f)\right] } & =0, & {[\mathbf{y}(h), \mathbf{z}(f)] } & =\mathbf{y}\left(\frac{2}{3} f^{\prime} h-f h^{\prime}\right) \\
{\left[\mathbf{x}\left(g_{1}\right), \mathbf{x}\left(g_{2}\right)\right] } & =0 & {\left[\mathbf{y}\left(h_{1}\right), \mathbf{y}\left(h_{2}\right)\right] } & =\mathbf{x}\left(\left(h_{1} h_{2}^{\prime}-h_{1}^{\prime} h_{2}\right) / 2\right) \\
{\left[\mathbf{z}\left(f_{1}\right), \mathbf{z}\left(f_{2}\right)\right] } & =\mathbf{z}\left(f_{1} f_{2}^{\prime}-f_{1}^{\prime} f_{2}\right) . & &
\end{aligned}
$$

These commutation relations show that a Kac-Moody-Virasoro (KMV) structure can be associated with an infinite dimensional subalgebra of $L[\underline{5}$, and the latter 
property tends to associate integrability with the $(2+1)$-dimensional ZK equation [13, 14, 15, which as is well-known can be linearized by a generalized hodograph transformation [16.

In order to classify subalgebras of $L$ under the adjoint action of its Lie group $\mathcal{G}$, we need to have an explicit expression for $\mathbf{w}(\epsilon)=\operatorname{Ad}(\exp (\epsilon \mathbf{v})) \mathbf{w}_{0}$, for every pair of generators $\mathbf{v}, \mathbf{w}_{0}$ of $L$. However, using the commutation relations (2.2) such an expression can easily be obtained either by interpreting $\mathbf{w}(\epsilon)$ as the flow of $\mathrm{Ad}$ through $\mathbf{w}_{0}$ of the one-parameter subgroup generated by $\mathbf{v}$, or again by rewriting $\mathbf{w}(\epsilon)$ in terms of the Lie series (see [17, P. 205]). The required classification of subalgebras of $L$ under the adjoint action of $\mathcal{G}$ can henceforth be achieved by applying known techniques [13, 17, 18. In this way, all one-dimensional and twodimensional subalgebras of $L$ were classified in 5 and for the sake of completeness as already mentioned, we present again in this paper not only the list of canonical forms of non-equivalent two-dimensional subalgebras of $L$ required for reductions to ODEs in which we are interested, but also the corresponding list for one-dimensional subalgebras. While the classification list for one-dimensional subalgebras does not contain free parameters, in the list for two-dimensional subalgebras, the parameters $k_{0}, k_{1}, c_{1}, c_{2}$, and $c_{3}$ are entirely free, unless otherwise stated. The classification lists are given as follows, where we have used the notation $\mathscr{C}_{n, j}, n=1,2$, instead of $\mathscr{L}_{n, j}$ as in the original list of [5], to represent the $j$ th canonical form of $n$-dimensional subalgebras of $L$.

(1) One-dimensional subalgebras

$\mathscr{C}_{1,1}=\{\mathbf{x}(1)\}, \quad \mathscr{C}_{1,2}=\{\mathbf{y}(1)\}, \quad \mathscr{C}_{1,3}=\left\{\mathbf{v}_{0}\right\}, \quad \mathscr{C}_{1,4}=\left\{k_{0} \mathbf{v}_{0}+\mathbf{z}(1)\right\}$

(2) Abelian two-dimensional subalgebras

$$
\begin{aligned}
\mathscr{C}_{2,1} & =\left\{k_{1} \mathbf{v}_{0}+\mathbf{z}(1), k_{0} \mathbf{v}_{0}+\mathbf{x}\left(c_{1} e^{2 k_{1} t}\right)+\mathbf{y}\left(c_{2} e^{k_{1} t}\right)\right\} \\
\mathscr{C}_{2,2} & =\{\mathbf{x}(1), \mathbf{x}(g)\}, \quad\left(g^{\prime} \neq 0\right) \\
\mathscr{C}_{2,3} & =\{\mathbf{x}(1), \mathbf{y}(h)\} \\
\mathscr{C}_{2,4} & =\left\{\mathbf{x}(1), k_{0} \mathbf{v}_{0}+\mathbf{z}\left(6 k_{0} t+c_{3}\right)\right\}, \quad k_{0}^{2}+c_{3}^{2} \neq 0 \\
\mathscr{C}_{2,5} & =\{\mathbf{y}(1), \mathbf{x}(g)\}, \quad\left(g^{\prime} \neq 0\right) \\
\mathscr{C}_{2,6} & =\left\{\mathbf{y}(1), k_{0} \mathbf{v}_{0}+\mathbf{z}\left((-3 / 2)\left(k_{0} t+c_{3}\right)\right)\right\}, \quad k_{0}^{2}+c_{3}^{2} \neq 0
\end{aligned}
$$

(3) Non abelian two-dimensional subalgebras

$$
\begin{aligned}
\mathscr{C}_{2,7} & =\left\{\mathbf{z}(1), k_{0} \mathbf{v}_{0}+\mathbf{x}\left(c_{1}\right)+\mathbf{y}\left(c_{2}\right)+\mathbf{z}(t)\right\} \\
\mathscr{C}_{2,8} & =\left\{\mathbf{x}(1), \mathbf{v}_{0}\right\} \\
\mathscr{C}_{2,9} & =\left\{\mathbf{x}(1), k_{0} \mathbf{v}_{0}+\mathbf{z}\left(\left(3-6 k_{0}\right) t+c_{3}\right)\right\}, \quad\left(k_{0}-\frac{1}{2}\right)^{2}+c_{3}^{2} \neq 0 \\
\mathscr{C}_{2,10} & =\left\{\mathbf{y}(1), \mathbf{v}_{0}\right\} \\
\mathscr{C}_{2,11} & =\left\{\mathbf{y}(1), k_{0} \mathbf{v}_{0}+\mathbf{z}\left((3 / 2)\left(1-k_{0}\right) t+c_{3}\right)\right\}, \quad\left(k_{0}-1\right)^{2}+c_{3}^{2} \neq 0
\end{aligned}
$$

The actual list $\mathscr{C}_{2, j}$ of two-dimensional subalgebras of $L$ is a simplified list obtained from the original list $\mathscr{L}_{2, j}$ of $[5]$. Indeed, the canonical form

$$
\mathscr{L}_{2,1}=\left\{\mathbf{v}_{0}, k_{0} \mathbf{v}_{0}+\mathbf{z}(1)\right\}
$$


can be obtained as a particular case of the Lie algebra $\mathscr{L}_{2,2}=\left\{Y_{1}, Y_{2}\right\}$, where

$$
Y_{1}=k_{1} \mathbf{v}_{0}+\mathbf{z}(1), \quad Y_{2}=k_{0} \mathbf{v}_{0}+\mathbf{x}\left(c_{1} e^{2 k_{1} t}\right)+\mathbf{y}\left(c_{2} e^{k_{1} t}\right)+\mathbf{z}\left(c_{3}\right),
$$

by setting $k_{0}=1, c_{j}=0$, for $j=1,2,3$ in $\mathscr{L}_{2,2}$. This reduces the list of canonical forms from 12 to 11 subalgebras, and in the new list, $\mathscr{L}_{2,2}$ becomes the first canonical form of two-dimensional subalgebras. For this reason, in order to avoid any confusion between representatives from the two lists, Lie algebras in the simplified list are denoted by $\mathscr{C}_{n, j}$, instead of $\mathscr{L}_{n, j}$. Further simplifications are obtained in the original list by eliminating some unnecessary parameters by a change of basis. For instance, the subalgebra $\mathscr{C}_{2,1}=\left\{V_{1}, V_{2}\right\}$ in the list above is obtained from the corresponding subalgebra $\mathscr{L}_{2,2}=\left\{Y_{1}, Y_{2}\right\}$ by setting $V_{1}=Y_{1}$, and $V_{2}=-c_{3} Y_{1}+Y_{2}$, which reduces to zero the parameter $c_{3}$ of $Y_{2}$, and this type of simplifications was often neglected in 5 .

\section{Similarity REDUCTIONS}

Similarity reductions of the ZK equation by symmetry subgroups whose actions are semi-regular with orbits of dimension $s$, where $0 \leq s<p$, and where $p=3$ is the number of independent variables, will yield equations in $s$ fewer independent variables. Each of the canonical forms $\mathscr{C}_{n, j}$ of one- and two-dimensional subalgebras of $L$ turns out to have $n$ dimensional orbits, and hence allows a reduction of the equation into one with $n$ fewer independent variables, provided certain regularity conditions are satisfied. In particular, reductions by one-dimensional subalgebras all yield $(1+1)$-dimensional reduced equations, while reductions by two-dimensional subalgebras yield ODEs. The usual procedure for this reduction is well known, and consists in the actual case where $\mathcal{G}$ acts projectably [17] in the space of independent and dependent variables, in finding invariants

$$
z=z(t, x, y), \quad w=w(t, x, y, u)
$$

of the corresponding subgroup action, where $z=\left(z_{1}, \ldots, z_{p-n}\right)$ has $p-n$ components, and to consider $z$ as the new set of independent variables and $w$ as the new dependent variable for the reduced equation. As usual, each reduction will be completely described by giving a reduction formula consisting in an expression for $z$ and for $u=u(t, x, y, w(z))$, and by providing the corresponding reduced equation.

3.1. Reduction by one-dimensional subalgebras. Reductions of the ZK equation based on canonical forms of one-dimensional subalgebras listed in (2.3) have already been performed in [5] as mentioned earlier, and the result repeated here for the sake of ensuring that the paper is as much self-contained as possible, is as follows in terms of the two components $r=z_{1}$ and $s=z_{2}$ of $z$.

(1) Reduction by $\mathscr{C}_{1,1}=\{\mathbf{x}(1)\}$.

For $\mathbf{x}(1)=\partial_{x}$, we simply have $u=u(t, y), r=t$, and $s=y$. The reduced equation is the linear equation

$$
u_{y, y}=0
$$

with solution $u=q_{1} y+q_{2}$, where $q_{1}$ and $q_{2}$ are arbitrary functions of $t$.

(2) Reduction by $\mathscr{C}_{1,2}=\{\mathbf{y}(1)\}$.

For $\mathbf{y}(1)=\partial_{y}$, we have $u=u(t, x), r=t, s=x$. The reduced equation is

$$
-u_{x}^{2}+u_{t, x}-u u_{x, x}=0 .
$$


(3) Reduction by $\mathscr{C}_{1,3}=\left\{\mathbf{v}_{0}\right\}$.

We have

$$
u=x w(t, s), \quad r=t, \quad s=y^{2} / 2,
$$

and the corresponding reduced equation is

$$
w_{r}-2 w_{s}-\left(w-s w_{s}\right)^{2}-s w_{r, s}-4 s w_{s, s}-s^{2} w w_{s, s}=0 .
$$

(4) Reduction by $\mathscr{C}_{1,4}=\left\{k_{0} \mathbf{v}_{0}+\mathbf{z}(1)\right\}$.

We have

$$
u=x w(r, s), \quad r=2 k_{0} t-\ln (x), \quad s=y^{2} / x,
$$

which gives rise to the reduced equation

$$
\begin{gathered}
2 w_{s}+\left(-w+w_{r}+s w_{s}\right)^{2}+2 k_{0}\left(w_{r, r}+s w_{r, s}\right)+4 s w_{s, s} \\
+w\left(-w_{r}+w_{r, r}+s\left(2 w_{r, s}+s w_{s, s}\right)\right)-2 k_{0} w_{r}=0 .
\end{gathered}
$$

For $k_{0}=0$, this last equation corresponds to the much simpler reduction by $\{\mathbf{z}(1)\}$ given by

$$
u_{x}^{2}+u u_{x, x}+u_{y, y}=0 .
$$

3.2. Reduction by two-dimensional subalgebras. To find the required similarity reductions, we shall treat each of the eleven canonical forms $\mathscr{C}_{2, j}$ separately. In this case $z$ has only one component and can be considered as the new independent variable. The parameters $k_{0}, k_{1}, c_{1}, c_{2}$, and $c_{3}$, if any, that appear in this reduction procedure are the same as those appearing in the corresponding canonical form $\mathscr{C}_{2, j}$ given in the previous section.

\subsubsection{Reduction by abelian subalgebras.}

(1) Reduction by $\mathscr{C}_{2,1}$. In this case the transversality condition on similarity variables does not hold for $c_{2}=0$, and we shall therefore assume that $c_{2} \neq 0$. We have

$z=-\frac{4 c_{1} c_{2} e^{k_{1} t} y+2 c_{1} y^{2} k_{0}+c_{2}^{2}\left(-4 x+y^{2} k_{1}\right)}{4\left(c_{2} e^{k_{1} t}+y k_{0}\right)^{2}}$

$u=\frac{-y k_{1}\left(8 c_{1} c_{2} e^{t k_{1}}+4 c_{1} y k_{0}+c_{2}^{2} y k_{1}\right)+4\left(c_{2} e^{k_{1} t}+y k_{0}\right)^{2} w(z)}{4 c_{2}^{2}}$

and the corresponding reduced equation is given by

$$
\begin{aligned}
& 4 c_{1} k_{0} k_{1}+c_{2}^{2} k_{1}^{2}-4 k_{0}^{2} w+\left(2 c_{1} k_{0}+4 z k_{0}^{2}+c_{2}^{2} k_{1}\right) w^{\prime} \\
& -2 c_{2}^{2} w^{\prime 2}+\left(-2 c_{1}^{2}-8 c_{1} k_{0} z-8 k_{0}^{2} z^{2}-4 c_{2}^{2} z k_{1}-2 c_{2}^{2} w\right) w^{\prime \prime}=0
\end{aligned}
$$

A much tractable case of (3.6) obtained by setting $c_{1}=k_{0}=0$ is given by

$$
k_{1}^{2}+k_{1} w^{\prime}-2 w^{2}-2\left(2 k_{1} z+w\right) w^{\prime \prime}=0,
$$

and as explained in Section 4.1, (3.6) turns out to have as a special case the equation

$$
c_{2}^{2} w^{\prime 2}+\left(c_{1}^{2}+c_{2}^{2} w\right) w^{\prime \prime}=0,
$$

obtained by setting $k_{0}=k_{1}=0$. Another case of (3.6) that we shall study is the equation

$$
-w^{2}+2(w z-1) w^{\prime}-z^{2} w^{2}-\left(4 z+w z^{2}\right) w^{\prime \prime}=0,
$$


obtained by setting $k_{0}=1$ and $k_{1}=c_{j}=0$ for $j=1,2$ in (3.6).

(2) Reduction by $\mathscr{C}_{2,2}$. In this case, the similarity variables $z$ and $w$ are given by $z=t, w=y$. Hence the corresponding Lie point transformation does not satisfy the transversality condition, since the equation $w(z)=y$ cannot be solved for $u$, and there is no reduced equation in this case.

(3) Reduction by $\mathscr{C}_{2,3}$. We have

$$
z=t, \quad u=\left(w(z)-y^{2}\right) h^{\prime \prime} / 4 h .
$$

In this case the reduced equation degenerates simply into the condition $h^{\prime \prime}=0$, which in turn yields only the trivial solution $u=0$.

(4) Reduction by $\mathscr{C}_{2,4}$. We have

$$
\begin{aligned}
& z=\frac{\left(c_{3}+6 k_{0} t\right)^{5}}{y^{6}}, \quad u=\frac{w(z)}{\left(c_{3}+6 k_{0} t\right)^{(1 / 3)}}, \quad \text { for } k_{0} \neq 0 \\
& z=y, \quad u=w(z), \quad \text { for } k_{0}=0
\end{aligned}
$$

and the corresponding reduced equation is linear and given by

$$
\begin{aligned}
7 w^{\prime}+6 z w^{\prime \prime} & =0, & & \text { for } k_{0} \neq 0 \\
w^{\prime \prime} & =0, & & \text { for } k_{0}=0 .
\end{aligned}
$$

(5) Reduction by $\mathscr{C}_{2,5}$. We have

$$
z=t, \quad u=g^{\prime}(w(z)-x / g) .
$$

Here again the reduced equation is simply a degenerated condition of the form $g^{\prime \prime}=0$, which gives rise to a solution of the form

$$
u=\lambda_{1}\left(w(t)-\frac{x}{\lambda_{1} t+\lambda_{2}}\right)
$$

where $\lambda_{1}$ and $\lambda_{2}$ are arbitrary constants, while $w(t)$ is an arbitrary function of $t$.

(6) Reduction by $\mathscr{C}_{2,6}$. We have

$z=x\left(c_{3}+k_{0} t\right), \quad u=w(z) /\left(c_{3}+k_{0} t\right)^{2}, \quad\left(k_{0}^{2}+c_{3}^{2} \neq 0\right)$,

and this leads to the reduced equation

$$
k_{0} w^{\prime}+w^{2}+\left(w-z k_{0}\right) w^{\prime \prime}=0 .
$$

\subsection{Reduction by non abelian subalgebras.}

(7) Reduction by $\mathscr{C}_{2,7}$. The reduction formula in this case is

$$
\begin{aligned}
& z=\frac{\left(6 k_{0}+1\right) x+3 c_{1}}{\left(\left(3 k_{0}+2\right) y+3 c_{2}\right)^{s}}, \quad u=\left(\left(6 k_{0}+1\right) x+3 c_{1}\right)^{r} w(z), \quad\left(k_{0} \neq-\frac{1}{6},-\frac{2}{3}\right) \\
& z=\frac{e^{x / c_{1}}}{\left(y+2 c_{2}\right)^{2}}, \quad u=\frac{w(z)}{e^{x / c_{1}}}, \quad\left(k_{0}=-1 / 6\right) \\
& z=\left(c_{1}-x\right) e^{y / c_{2}}, \quad u=\left(c_{1}-x\right)^{2} w(z), \quad\left(k_{0}=-2 / 3\right)
\end{aligned}
$$


where $s=\left(6 k_{0}+1\right) /\left(3 k_{0}+2\right)$ and $r=\left(6 k_{0}-2\right) /\left(6 k_{0}+1\right)$. This leads to the following reduced equation when $k_{0} \neq-1 / 6$ and $k_{0} \neq-2 / 3$.

$$
\begin{aligned}
&-r(-1+2 r) w^{2} z^{r}\left(1+6 k_{0}\right)^{2}+\left(-s(1+s) z^{3}\left(2+3 k_{0}\right)^{2}\right. \\
&\left.-4 r w z^{r+1}\left(1+6 k_{0}\right)^{2}\right) w^{\prime}-z^{r+2}\left(1+6 k_{0}\right)^{2} w^{\prime 2} \\
&+\left(-s^{2} z^{4}\left(2+3 k_{0}\right)^{2}-w z^{r+2}\left(1+6 k_{0}\right)^{2}\right) w^{\prime \prime}=0 .
\end{aligned}
$$

The other reduced equations for $k_{0}=-1 / 6$ and $k_{0}=-2 / 3$ are respectively given by

$$
\begin{aligned}
-2 w^{2}+\left(3 w z-6 c_{1}^{2} z^{2}\right) w^{\prime}-z^{2} w^{\prime 2}+\left(-w z^{2}-4 c_{1}^{2} z^{3}\right) w^{\prime \prime} & =0, \\
-6 c_{2}^{2} w^{2}-\left(z+8 c_{2}^{2} w z\right) w^{\prime}-c_{2}^{2} z^{2} w^{\prime 2}-\left(z^{2}+c_{2}^{2} w z^{2}\right) w^{\prime \prime} & =0,
\end{aligned}
$$

(8) Reduction by $\mathscr{C}_{2,8}$. We have

$$
z=t, \quad u=y^{2} w(z),
$$

which only results in the trivial equation $w=0$, and solution $u=0$.

(9) Reduction by $\mathscr{C}_{2,9}$. We have

$$
\begin{array}{lll}
z=\frac{c_{3}+\left(3-6 k_{0}\right) t}{y^{r}}, & u=\left(c_{3}+\left(3-6 k_{0}\right) t\right)^{s} w(z), & \text { for } k_{0} \neq 1 / 2 \\
z=e^{\left(-t / 2 c_{3}\right)} y, & u=e^{t / c_{3}} w(z), & \text { for } k_{0}=1 / 2 .
\end{array}
$$

where

$$
r=\left(3-6 k_{0}\right) /\left(2-3 k_{0}\right) \text { and } s=\left(6 k_{0}-2\right) /\left(3-6 k_{0}\right),
$$

and this leads to the linear reduced equation

$$
\begin{aligned}
\left(-5+9 k_{0}\right) w^{\prime}+3 z\left(-1+2 k_{0}\right) w^{\prime \prime} & =0, & & \text { for } k_{0} \neq 1 / 2 \\
w^{\prime \prime} & =0, & & \text { for } k_{0}=1 / 2
\end{aligned}
$$

(10) Reduction by $\mathscr{C}_{2,10}$. We have

$$
z=t, \quad u=x w(z),
$$

and this gives rise to the reduced equation

$$
w^{2}-w^{\prime}=0
$$

(11) Reduction by $\mathscr{C}_{2,11}$. We have

$$
\begin{aligned}
& z=\left(3\left(1-k_{0}\right) t+2 c_{3}\right) / x^{s}, \quad u=\left(3\left(1-k_{0}\right) t+2 c_{3}\right)^{r} w(z), \quad \text { for } k_{0} \neq 1,-1 / 3 \\
& z=e^{\left(2 t / c_{3}\right)} / x, \quad u=e^{\left(2 t / c_{3}\right)} w(z), \quad \text { for } k_{0}=1 \\
& z=x, \quad u=w(z) /\left(c_{3}+2 t\right), \quad \text { for } k_{0}=-1 / 3
\end{aligned}
$$

where $s=3\left(1-k_{0}\right) /\left(1+3 k_{0}\right)$ and $r=\left(2\left(3 k_{0}-1\right)\right) /\left(3\left(1-k_{0}\right)\right)$. This yields for $k_{0} \neq 1,-1 / 3$, the reduced equation

$\left[3(1+r)\left(k_{0}-1\right)-(1+s) w z^{1+r}\right] w^{\prime}-s z^{2+r} w^{2}+\left(3 z\left(k_{0}-1\right)-s w z^{2+r}\right) w^{\prime \prime}=0$

while the other reduced equations are

$$
\begin{aligned}
\left(4+2 c_{3} w z\right) w^{\prime}+c_{3} z^{2} w^{\prime 2}+z\left(2+c_{3} w z\right) w^{\prime \prime} & =0, & & \text { for } k_{0}=1 \\
2 w^{\prime}+w^{\prime 2}+w w^{\prime \prime} & =0, & & \text { for } k_{0}=-1 / 3
\end{aligned}
$$


All of the reduced ODEs that we have thus obtained for the ZK equation belong to a more general family of differential equations of the form

$$
A_{1}+A_{2} w^{\prime}+A_{3} w^{2}+A_{4} w^{\prime \prime}=0
$$

where the $A_{j}$ are polynomials in $w$ whose coefficients are functions of $z$, and of the form

$$
\begin{aligned}
& A_{1}=\alpha_{1} w^{2}+\alpha_{2} w z^{r}+\alpha_{3} w z^{3}+\alpha_{4} z^{3}+\alpha_{5} \\
& A_{2}=\beta_{1} w z^{r+1}+\beta_{2} w z+\beta_{3} w+P_{4}(z) \\
& A_{3}=\gamma_{1} z^{r+2}+P_{2}(z) \\
& A_{4}=\delta_{1} w z^{r+2}+\delta_{2} w z^{2}+\delta_{3} w z+\delta_{4} w+P_{5}(z),
\end{aligned}
$$

where the $\alpha_{j}, \beta_{j}, \gamma_{j}, \delta_{j}$ and $r$ are constants, while $P_{n}(z)$ represents a polynomial of degree $n$ in $z$. These equations are generally nonlinear, except in the case of reductions by $\mathscr{C}_{2,4}$ and $\mathscr{C}_{2,9}$ where the corresponding equations of the form (3.29) turn out to be genuine linear equations of the simple form

$$
a_{1} w^{\prime}+a_{2} z w^{\prime \prime}=0,
$$

where $a_{1}$ and $a_{2}$ are some functions of $z$.

\section{ExaCt SOlutions AND THEIR PROPERTIES}

As already mentioned, we shall be interested in this paper only in solutions resulting from the reduced ODEs, and in so doing we shall pay a special attention to bounded solutions of the ZK equation. It is indeed well-known that bounded sound beams have important applications in underwater acoustics [19, and in medicine [20, and a whole chapter of [21] is devoted to a discussion of nonlinear bounded sound waves. Bounded solutions of the ZK equation are solutions which are bounded functions of the spatial variable $x$ aligned with the primary direction of propagation, and the time-like variable $t$. In this sense, not all solutions of the ZK equation are bounded, and this is the case with the solution given for example by (3.14). In the sequel, $\lambda$ and $\lambda_{j}$, for $j=1,2$, will always represent arbitrary constants of integration.

The analysis of the previous section shows that reduced ODEs are obtained as linear equations only in (3.12) and (3.23). In the case of (3.12), it is readily seen that solving (3.12a) and reverting back yields the corresponding solution

$$
u=\frac{-6 \lambda_{1} y+\lambda_{2}}{\sqrt{c_{3}+6 k_{0} t}},
$$

and this is clearly a bounded solution in the above stated sense. Similarly, it is readily seen that the reduced equation (3.12b yields the solution $u=\lambda_{1} e^{t / c_{3}}+$ $\lambda_{2} e^{t /\left(2 c_{3}\right)} y$ of the original equation, which is bounded only if $c_{3}$ is a negative number. For (3.23), solving the reduced equation (3.23a) and applying (3.21a) yields the solution

$$
u=X^{s}\left[\frac{\lambda_{1}\left(\left(3-6 k_{0}\right) X\right)^{-1 / r} y}{-2+3 k_{0}}+\lambda_{2}\right], \quad X=c_{3}+\left(3-6 k_{0}\right) t,
$$


in which $r$ and $s$ are constants given by (3.22), and which in the actual case determine when the solution (4.2) is bounded. On the other hand, it is readily seen that the solution of the ZK equation corresponding to (3.23b is $u=$ $e^{t / c_{3}}\left(\lambda_{1}+\lambda_{2} e^{-t /\left(2 c_{3}\right)} y\right)$, which is bounded for $c_{3}<0$.

For nonlinear reduced equations, the simplest and most frequent equations of the form (3.29) are equations in which the coefficients $A_{j}$ are simple polynomial functions in $w$ and $z$. More precisely, these are given by $A_{1}=\alpha w^{2}$, and $A_{3}=\beta z^{2}$, where $\alpha$ and $\beta$ are constants, while $A_{2}$ and $A_{4}$ are linear in $w$. We call Type $A$ this class of simpler reduced nonlinear ODEs, and Type $B$ its complement among the nonlinear reduced ODEs. Type $A$ equations often correspond to subcases of Type $B$ equations which in turn correspond to general cases of reductions by $\mathscr{C}_{2,1}, \mathscr{C}_{2,7}$ and $\mathscr{C}_{2,11}$. We can partition Type $A$ into Type $A^{1}$ consisting of equations admitting only one symmetry, and Type $A^{2}$, consisting of equations admitting two or more symmetries. All Type $A$ equations can generally be reduced to an Abel equation of the first kind, which as is well-known are generally difficult to solve [23, 24]. However, when any such equation has two known symmetries, it is possible to obtain solutions of the nonlinear equation, at least in implicit form. We will illustrate this point by treating a number of examples.

If we consider for instance Type $A^{1}$ reduced equation (3.9) obtained as a reduction by a particular case of $\mathscr{C}_{2,1}$, we observe that this equation has only a one-dimensional symmetry algebra generated by

$$
\mathbf{v}=z \partial_{z}-w \partial_{w}
$$

with rectifying coordinates $r=w z$ and $Y=-\ln (w)$. In terms of these new variables, (3.9) takes the form

$$
-1+(2-5 r) Y^{\prime}+\left(-8 r+11 r^{2}\right) Y^{\prime 2}-6 r^{2}(1+r) Y^{\prime 3}+r(4+r) Y^{\prime \prime}=0,
$$

which is clearly an equation of Abel of the first kind for $Z=Y^{\prime}$. However, even the transformed equation (4.4) also has only one symmetry, and thus it is difficult to find its solutions by Lie groups methods. Similar results apply to essentially all other Type $A^{1}$ reduced ODEs such as (3.16) and (3.19).

Among Type $A^{2}$ equations, one of the simplest is (3.25), which is of the first order and corresponds to a reduction by $\mathscr{C}_{2,10}$, but the corresponding solution $u=$ $x /(\lambda-t)$ to (1.2) is not a bounded one. In all other Type $A^{2}$ cases, the equation is of order two and can also be reduced to an Abel equation of the first kind. A simple example for this case is (3.28b). It admits the symmetry $\mathbf{v}=\partial_{z}$, in terms of whose rectifying variables $r=w, Y=z$, the equation takes the form

$$
2 Y^{\prime 2}+Y^{\prime}-r Y^{\prime \prime}=0
$$

and thus reduces to a Riccati equation for $Z=Y^{\prime}$, which is just a degenerated form of an Abel equation. If we now set $Y^{\prime}=-r /(V+1)$, then (4.5) is transformed to the linear equation $V^{\prime}-2=0$. Solving this last equation and reverting back step by step shows that (3.28b) has solution

$$
w=-\frac{1}{2} A\left[1+W\left(-\exp \left(\frac{-A+4(z-B)}{A}\right) / A\right)\right],
$$


and the corresponding solution to the ZK equation is given by

$$
\begin{aligned}
& u=-\frac{A}{2\left(c_{3}+2 t\right)}\left[1+W\left(-\exp \left(\frac{-A+4(x-B)}{A}\right) / A\right)\right], \quad A \neq 0 \\
& u=2(B-x) /\left(c_{3}+2 t\right), \quad A=0
\end{aligned}
$$

where $A$ and $B$ are arbitrary constants, and where $W(z)$ is the inverse of the function $w \mapsto w e^{w}=z$, and is called Lambert function [22].

For $z$ real, $W(z)$ is a real-valued function defined only for $z \geq-1 / e$, and it has two branches on $(-1 / e, 0)$. The branch satisfying $-1 \leq W(z)$ is denoted $W_{0}(z)$, and called the principal branch of $W$, or again the product log. The other branch satisfying $W(z) \leq-1$ is denoted by $W_{-1}(z)$, and decreases from $W_{-1}(-1 / e)=-1$ to $W\left(0^{-}\right)=-\infty$. When $z$ is complex, $W(z)$ takes on complex values and has an infinity of branches denoted by $W_{k}, k \in \mathbb{Z}$. More information on the complex analysis, numerical analysis, asymptotics, and symbolic calculus of the Lambert function can be found in 22. and the references therein, which also contain discussions on the algebraic properties and the many applications of this special function .

Since all the variables in the ZK equation are assumed to be real, (4.7a) represents a real function only if

$$
x \leq(A \ln (A)+4 B) / 4 \quad \text { for } A>0, \quad \text { and } \quad x \in(-\infty,+\infty) \quad \text { for } A<0 .
$$

When the latter condition (4.8) is satisfied, the corresponding solution (4.7) with $W(z)=W_{0}(z)$ is always bounded for $A>0$. For $A<0$ it is bounded only if we assume $x>0$, and it is not bounded for $A=0$.

Equation (3.28a has the same properties, although it is less obvious to solve. It has two symmetries, $\mathbf{v}=z^{2} \partial_{z}+\left(2 / c_{3}\right) \partial_{w}$ and $\mathbf{w}=z \partial_{z}-w \partial_{w}$, which satisfy a commutation relation of the form $[\mathbf{w}, \mathbf{v}]=\mathbf{v}$. Thus by the well-know procedure of [17, the equation should first be reduced by the normal symmetry subalgebra generated by $\mathbf{v}$. Before trying to solve (3.28a), we notice that in terms of the rectifying coordinates $r=2 / z+c_{3} w$ and $Y=3 c_{3} w / 2+2 / z$ of $\mathbf{v}$, (3.28a) takes the form

$$
\frac{6}{r}-\frac{16}{r} Y^{\prime}+\frac{14}{r} Y^{\prime 2}-\frac{4}{r} Y^{\prime 3}+Y^{\prime \prime}=0,
$$

which is clearly an Abel equation for $Z=Y^{\prime}$. On the other hand, in terms of the invariants $\xi=2 / z+c_{3} w$ and $X=z^{2} w^{\prime}$ of the first prolongation of $\mathbf{v}$, 3.28a reduces to the first order equation

$$
-c_{3} X^{2}+\xi\left(2-c_{3} X\right) X^{\prime}=0 .
$$

In terms of the new variables $\xi$ and $X$, w reduces in the quotient space $\mathcal{G} / \mathcal{H}$, where $\mathcal{H}$ is the one-parameter subgroup generated by $\mathbf{v}$, to a symmetry $\tilde{\mathbf{w}}=-\xi \partial_{\xi}$ of the reduced equation. Finally, when expressed in terms of the rectifying coordinates $v=X$ and $Q=-\ln (\xi)$ of $\tilde{\mathbf{w}}$, (4.10) reduces to

$$
-2+c_{3} v-c_{3} v^{2} Q^{\prime}=0,
$$

with solution

$$
Q=A+\frac{2}{c_{3} v}+\ln (v)
$$

where $A$ is an arbitrary constant of integration. However, trying to revert back to the solution $w(z)$ to the original equation (3.28a) only leads to an implicit expression 
for $w(z)$ of the form

$$
w-\frac{2+c_{3} z w}{c_{3} z W(f(z, w))}=B
$$

where

$$
f(z, w)=-2 e^{\left(-A / c_{3}\right)}\left(2+c_{3} z w\right) /\left(c_{3} z\right),
$$

and where $A$ and $B$ are the two arbitrary constants of integration. Consequently, it is not obvious how to determine the corresponding solution $u$ to the ZK equation and its properties from (4.12). Similar difficulties are encountered with all Type $B$ reduced equations, which turn out to have only zero- or one-dimensional symmetry algebras, and which by essence are more complicated then the Type $A$ ones we have considered.

Reductions by type $\mathscr{C}_{2,1}$ Lie subalgebras appear to be the only cases of reductions by two-dimensional subalgebras which provide invariant solutions that might describe sound wave propagation depending on both spatial variables $x$ and $y$, and time $t$. A particular case of such a reduction is given by (3.7), whose symmetry algebra $L_{2 a}$ is found after some long calculations to be generated by

$$
\begin{aligned}
& V_{1}=\partial_{z}-2 k_{1} \partial_{w}, \quad V_{2}=z \partial_{z}+w \partial_{w} \\
& V_{3}=\frac{1}{2}\left(w-k_{1} z\right)\left(w-5 k_{1} z\right) \partial_{z}+k_{1}\left(w-k_{1} z\right)\left(2 w+k_{1} z\right) \partial_{w},
\end{aligned}
$$

with commutation relations

$$
\left[V_{1}, V_{2}\right]=V_{1}, \quad\left[V_{1}, V_{3}\right]=-9 k_{1}^{2} V_{2}, \quad\left[V_{2}, V_{3}\right]=V_{3},
$$

which show that $L_{2 a}$ is a semisimple Lie algebra. In terms of the invariants

$$
v=\frac{-2 k_{1} \alpha_{1}+\alpha_{2} w}{\alpha_{1}+\alpha_{2} z}, \quad H=w^{\prime}(z)
$$

of the first prolongation of $\alpha_{1} V_{1}+\alpha_{2} V_{2}$, where $\alpha_{1}, \alpha_{2}$ are some constants, (3.7) is reduced to

$$
k_{1}^{2}-2 H^{2}+2 v\left(v+2 k_{1}\right) H^{\prime}+H\left(k_{1}-2\left(v+2 k_{1}\right) H^{\prime}\right)=0 .
$$

Solving this last equation and reverting back shows that the corresponding solutions to the original ZK equation are given by

$$
\begin{aligned}
u= & \frac{3 e^{2 k_{1} t} \alpha_{1} k_{1}}{\alpha_{2}}+k_{1} x-\frac{y^{2} k_{1}^{2}}{2}, \text { or } \\
u= & -\frac{1}{2 \alpha_{2}^{2}}\left[\alpha_{2}^{2} k_{1}\left(-2 x+y^{2} k_{1}\right)-6 e^{2 k_{1} t} \alpha_{2} k_{1}\left(\alpha_{1}+\imath e^{\lambda_{2}+\frac{2 \lambda_{1}}{9}} k_{1} \alpha_{2}\right)\right] \\
& -\frac{2 e^{2 k_{1} t}}{\alpha_{2}^{2}}\left[e^{\lambda_{2}} \alpha_{2}^{3} k_{1}\left(\alpha_{1}+\alpha_{2}\left(\imath e^{\lambda_{2}+\frac{2 \lambda_{1}}{9}} k_{1}+e^{-2 k_{1} t}\left(x-\frac{y^{2} k_{1}}{4}\right)\right)\right)\right],
\end{aligned}
$$

where $\lambda_{1}$ and $\lambda_{2}$ are two constants of integration. There are of course other invariant solutions of the ZK equation resulting from (3.7) that are too cumbersome to be mentioned here. On the other hand, neither these solutions, nor those indicated in (4.15) are bounded, although they all depend on both spatial variables $x, y$, and time $t$. 
Similar solutions can be obtained from (3.8), which is another case of reduction by $\mathscr{C}_{2,1}$, corresponding to $k_{0}=k_{1}=0$. Under the change of variables

$$
r=-1 / z, \quad H=\frac{1}{z}\left(w+\frac{c_{2}^{2}}{2 c_{1}^{2}} w^{2}+1\right)
$$

this equation is transformed into the free fall equation $H^{\prime \prime}=0$, and this gives rise to a solution of the ZK equation of the form

$$
u=\frac{-c_{1}^{2}+\varepsilon \sqrt{c_{1}^{2}\left(c_{1}^{2}-2 c_{2}^{2}\left(1+\lambda_{1}-\lambda_{2} x\right)-2 \lambda_{2} c_{1} c_{2} y\right)}}{c_{2}^{2}}, \quad \varepsilon \in\{-1,1\},
$$

for some constants of integration $\lambda_{1}$ and $\lambda_{2}$. However, this solution too is not a bounded one, and it turns out that bounded solutions to the ZK equation have occurred generally only in the case of linear reduced equations, or in terms of the Lambert function $W$.

4.1. Linearizability. Our analysis in this section has revealed that in general all nonlinear reduced ODEs of the ZK equation were not readily integrable. Most often this is due to the fact that their solutions is not expressible in terms of algebraic or elementary functions. Before attempting to solve nonlinear ODEs obtained by similarity reductions, it is customary to perform a painlevé test 25, 26] that determines whether these equations satisfy certain necessary conditions for having the so-called Painlevé property, because Painlevé-type equations are generally perceived to be easier to solve than general nonlinear ODEs. For a given differential equation, the Painlevé property is the property that all solutions of the equation are free from movable essential singularities, and for second order ODEs a list of fifty canonical forms of all such equations exists [27.

However, since we have attempted to solve all the nonlinear reduced ODEs without performing any Painlevé test on them, we will simply contempt ourselves with checking whether the nonlinear reduced equations obtained, each of which may be put into the form

$$
\Delta \equiv w^{\prime \prime}+F\left(z, w, w^{\prime}\right)=0,
$$

for a certain function $F$, are linearizable by a point transformation of the form

$$
z=f(H, r), \quad w=g(H, r) .
$$

This will give a better insight into the integrability of the ZK equation, because linear equations are by essence integrable. Indeed, in the modern era of integrability, the Painlevé approach has had an immense success by leading in particular to the discovery of a wealth of new integrable systems, to the extent that various conjectures almost intimately associated the Painlevé property with integrability 28. However, it has become clear that although many linearizable equations (both ordinary and partial) possess the Painlevé property, many other linearizable equations do not possess this property [28, 29]. By a result of Lie, the linearization test is easy to perform. Indeed, Lie [30] showed that a necessary condition for an equation of the form (4.18) to be linearizable is that it be of the form

$$
\Delta \equiv w^{\prime \prime}+A(z, w) w^{\prime 3}+B(z, w) w^{2}+C(z, w) w^{\prime}+D(z, w)=0,
$$


that is, a polynomial in $w^{\prime}$ of degree at most 3 . If for a given equation of the above form (4.20) we consider the two expressions

$$
\begin{aligned}
& \Psi_{1}(\Delta)=3 A_{z, z}-2 B_{z, w}+C_{w, w}-3(C A)_{z}+3(D A)_{w}+\left(B^{2}\right)_{z}+3 A D_{w}-B(C)_{w} \\
& \Psi_{2}(\Delta)=3 D_{w, w}-2 C_{z, w}+B_{z, z}-3(D A)_{z}+3(D B)_{w}-\left(C^{2}\right)_{w}-3 D A_{z}+C B_{z},
\end{aligned}
$$

then Lie also showed [30, 31] that an equation of the form (4.20) is linearizable if and only if

$$
\Psi_{1}(\Delta)=0, \quad \text { and } \quad \Psi_{2}(\Delta)=0,
$$

and he also provided a method for finding the linearizing transformations of the form (4.19).

All the nonlinear ODEs obtained for the ZK equation by similarity reductions are clearly of the form (4.20) required for the necessary condition of linearization. However, we have found that none of them is linearizable in general, except in some few cases where linearization is possible for a unique value of the set of parameters. Indeed, if for every equation $\Delta$ of the form (4.18) we set $\Psi(\Delta)=\left(\Psi_{1}(\Delta), \Psi_{2}(\Delta)\right)$, then it is readily seen that in the case of (3.16) for instance, we have $\Psi(\Delta)=0$ if and only if $k_{0}=0$ (and $c_{3} \neq 0$ ), and for this value of $k_{0}$ the equation reduces to

$$
w^{2}+w w^{\prime \prime}=0
$$

which transforms into the linear equation $Z^{\prime \prime}=0$ for $Z=w^{2}$. A similar nonlinearity property is observed about other Type $A$ equations containing parameters. Indeed, the test reveals that (3.8), (3.19), and (3.28) are not linearizable.

On the other hand none of the Type $A$ equations without parameters is linearizable. For instance for (3.9) we have

$$
\Psi(\Delta)=\left(\frac{54 z}{(4+w z)^{3}},-\frac{72(-2+w z)}{z(4+w z)^{3}}\right) .
$$

For Type $B$ equations which usually represent the most general types of reduced equations involving several parameters, and which are generally represented by (3.6), (3.18) and (3.27), linearization occurs at most for a fixed value of a set of parameters. Thus, (3.6) which is the most general type of equations corresponding to a reduction by $\mathscr{C}_{2,1}$ is linearizable if and only if $k_{0}=k_{1}=0$, and the linearizing transformations of the corresponding reduced equation (3.8) is given by (4.16). This explains why we referred earlier to (3.8) as a special case of (3.6). On the other hand, (3.18) is not linearizable while (3.27) is linearizable if and only if $k_{0}=1 / 9$. The linearization test thus shows that all nonlinear ODEs obtained by similarity reductions of the ZK equation are essentially non linearizable.

\section{Concluding Remarks}

Based on the classification list of all two-dimensional subalgebras of the $(2+1)$ dimensional ZK equation recently proposed in [5], and on the symmetry reduction methods of [17, we have obtained for the first time in this paper all possible similarity reductions to ODEs of the ZK equation (1.2). In this way, new exact solutions of this equation which are larger in number and different from those obtained in [3, 4, 6] have been derived.

In general, all nonlinear reduced equations were not readily integrable, and instead of performing the usual Painlevé test on these equations, we have rather shown that all nonlinear reduced equations are essentially non linearizable. Bounded 
solutions generally occurred only in the case of linear reduced equations, or in terms of the Lambert function. We've also obtained numerous exact solutions depending on both spatial variables $x, y$ and time $t$. For the sake of completeness we've also included in this exposition the list from [5] of canonical forms of onedimensional subalgebras of the ZK symmetry algebra, as well the corresponding reduced $(1+1)$-dimensional equations.

\section{REFERENCES}

[1] Zabolotskaya E A and Khokhlov R V 1969 Soviet Phys. Acoust. 1535

[2] Kuznetsov V P 1971 Sov. Phys. Acoust. 16467

[3] Vinogradov A M and Vorob'yev E M 1976 Akust. Zh. 2223

[4] Chowdhury A R and Nasker M 1986 J. Phys. A: Math. Gen. 191775

[5] Ndogmo J C 2008 Nonlinear Dyn. DOI 10.1007/s11071

[6] Zhang J F, Zhu Y J and Lin J 1995 Commun. Theor. Phys. 2469

[7] Webb G M and Zank G P 1990 J. Phys. A: Math. Gen. 235465

[8] Sindaoreji 1999 J. Phys. A: Math. Gen. 326897

[9] Soh Wafo C and Momoniat E 2004 IMA J. Appl. Math. 69219

[10] Güngör F 2001 J. Phys. A: Math. Gen. 344313

[11] Schwarz F 1987 J. Phys. A: Math. Gen. 201613

[12] Hereman W, Steeb W H and Euler N 1992 J. Phys. A: Math. Gen. 252417

[13] David D, Kamran N, Levi D and Winternitz P 1986 J. Math. Phys. 271225

[14] Winternitz P 1988 Kac-Moody-Virasoro, symmetries of integrable nonlinear partial differential equations Preprint CRM 1548

[15] Güngör F 2006 SIGMA 2 014, 7 pp

[16] Gibbons J and Kodama Y 1987 Proc. Int. Conf. on Nonlinear evolutions (Teaneck: World Sci. Publ.) P 97

[17] Olver P J 1986 Applications of Lie Groups to Differential Equations (New York: Springer)

[18] Winternitz P 1993 Lie groups and solutions of nonlinear partial differential equations Preprint CRM-1841

[19] Novikov B K, Rudenko O V and Timoshenko V I 1987 Nonlinear underwater acoustics (New York: American Institute of Physics)

[20] Averkiou M A and Cleveland R O 1999 J. Acoust. Soc. Am. 106102

[21] Enflo B O and Hedberg C M 2004 Theory of nonlinear acoustics in fluids (New York: Springer)

[22] Corless R M, Gonnet G H, Hare D E G, Jeffrey D J, and Knuth D E 1996 Adv. Comput. Math. 5329

[23] Polyanin A D and Zaitsev V F 1995 Hanbook of Exact solutions for ordinary differential Equations (Boca: CRC Press)

[24] Liouville R 1903 Acta Math. 2755

[25] Ablowitz M J, Ramani A and Segur H A 1980 J. Math. Phys. 21715

[26] Rand D W and Winternitz P 1986 Comput. Phys. Comm. 42359

[27] Ince E L 1956 Ordinary differential equations (New York: Dover)

[28] Tamizhmani K M, Grammaticos B and Ramani A 2007 SIGMA 3 073, 6pp

[29] Ramani A, Grammaticos B and Tremblay S 2000 J. Phys. A: Math. Gen 333045

[30] Lie S 1883 Archiv for Mathematik og Naturvidenskrab 8371

[31] Ibragimov N H and Magri F 2004 Nonlinear Dyn. 3641

PO Box 2446 Bellville 7535, South Africa,

E-mail address: ndogmoj@yahoo.com 